\title{
Rcor2 underexpression in senescent mice: a target for inflammaging?
}

\author{
María J Alvarez-López ${ }^{1,2}$, Patricia Molina-Martínez ${ }^{1}$, Marco Castro-Freire ${ }^{3}$, Marta Cosín-Tomás ${ }^{1,2}$, Rosa Cristòfol ${ }^{1}$, \\ Marcelina Párrizas ${ }^{3}$, Rosa María Escorihuela ${ }^{4}$, Merce Pallàs ${ }^{2}$, Coral Sanfeliu ${ }^{1,3}$ and Perla Kaliman ${ }^{3^{*}}$
}

\begin{abstract}
Background: Aging is characterized by a low-grade systemic inflammation that contributes to the pathogenesis of neurodegenerative disorders such as Alzheimer's disease (AD). However, little knowledge is currently available on the molecular processes leading to chronic neuroinflammation. In this context, recent studies have described the role of chromatin regulators in inflammation and longevity including the REST corepressor (Rcor)-2 factor, which seems to be involved in an inflammatory suppressive program.

Methods: To assess the impact of Rcor2 in age-related inflammation, gene expression levels were quantified in different tissues and ages of the spontaneous senescence-accelerated P8 mouse (P8) using the SAMR1 mouse (R1) as a control. Specific siRNA transfection in P8 and R1 astrocyte cultures was used to determine Rcor2 involvement in the modulation of neuroinflammation. The effect of lipopolysaccharide (LPS) treatment on Rcor2 levels and neuroinflammation was analyzed both in vivo and in vitro.

Results: P8 mice presented a dramatic decrease in Rcor2 gene expression compared with R1 controls in splenocytes, an alteration also observed in the brain cortex, hippocampus and primary astrocytes of these mice. Rcor2 reduction in astrocytes was accompanied by an increased basal expression of the interleukin (II)- 6 gene. Strikingly, intraperitoneal LPS injection in R1 mice downregulated Rcor2 in the hippocampus, with a concomitant upregulation of tumor necrosis factor (Tnf- $a$ ), $/ 17-\beta$ and $/ 16$ genes. A negative correlation between Rcor2 and $/ 16$ gene expression was also verified in LPS-treated C6 glioma cells. Knock down of Rcor2 by siRNA transfection (siRcor2) in R1 astrocytes upregulated $/ 16$ gene expression while siRcor2 further increased $/ 16$ expression in P8 astrocytes. Moreover, LPS activation provoked a further downregulation of Rcor2 and an amplified induction of 116 in siRcor2-tranfected astrocytes.
\end{abstract}

Conclusions: Data presented here show interplay between Rcor2 downregulation and increased inflammation and suggest that Rcor2 may be a key regulator of inflammaging.

Keywords: RCOR2, Aging, SAMP8, Inflammation, IL6

\section{Background}

The process of aging is associated with a low-grade systemic inflammation defined as inflammaging [1], which is characterized by an increase of acute-phase circulating proteins and proinflammatory factors such as interleukin (IL)-6 and tumor necrosis factor (TNF- $\alpha$ ). This condition has been connected with the onset and evolution of age-related diseases, including type 2 diabetes mellitus,

\footnotetext{
*Correspondence: pkaliman@ub.edu

${ }^{3}$ Instituto de Investigaciones Biomédicas August Pi i Sunyer (IDIBAPS),

Rosellón 149, E-08036 Barcelona, Spain

Full list of author information is available at the end of the article
}

cardiovascular disease, cancer and neurodegeneration [2-4]. Indeed, the aging brain exhibits a progressive increase in inflammatory processes [5], which can be neurotoxic, altering important central functions [6] and contributing to the pathogenesis of neurodegenerative disorders such as Alzheimer's disease (AD) [7]. The microglia plays a critical role in neuroinflammation as it represents the main type of immune cells in the brain [8]. The activation of microglia in aging models leads to the secretion of proinflammatory cytokines, which contribute to synaptic and neuronal damage as well as cognitive impairment $[9,10]$. 
Recent studies using diverse experimental models have described the role of chromatin regulators in inflammation. Among them, REST corepressor (RCOR) factor seems to be involved in an inflammatory suppressive program. In mammals, two Rcor gene isoforms (Rcor1/CoREST and Rcor2) have so far been studied. RCOR is found in a complex with the histone demethylase lysine-specific demethylase 1A (KDM1A, previously known as LSD1), which exhibits nucleosome demethylase activity [11-13], and with the RE1-silencing transcription factor (REST), which has been recently associated with cognitive preservation and longevity [14]. RCOR1/CoREST is involved in the modulation the expression of neuronal genes [15] while RCOR2 plays a role in embryonic stem cells (ESCs) maintenance and regulation [13]. We have recently shown that knock down of LSD1/KDM1A or its partner, RCOR2, in preadipocytes results in derepression of an inflammatory program with a significant increase in the expression of inflammatory genes such as Il6, chemokine ligand 2 (Ccl2), macrophage proinflammatory protein $1 \alpha$ (Mip1a, also known as $\mathrm{Ccl} 3$ ), chemokine (C-X-C motif) ligands 5 and 10 ( $\mathrm{Cxcl5}$ and $\mathrm{Cxcl10}$ ), and macrophage activation 2-like (Mpa2l) [16]. Consistent with these findings, RCOR was also found to mediate the turnover of nuclear factor kappa $\mathrm{B}(\mathrm{NF}-\mathrm{kB})$ and to restore LPS-activated genes to a basal expression in microglia and astrocytes [17].

Here, we examined the link between Rcor2 and inflammation in the SAMP8 mouse model of accelerated aging and neurodegeneration. The SAMP8 strain (P8) was selected from AKR/J mice and is a well-characterized model for studying pathological brain aging [18-21]. The SAMR1 mice (R1), with a similar genetic background and normal aging characteristics, represent a suitable and widely used control model [22]. P8 mice presented signs of accelerated aging such as loss of activity, skin coarseness, alopecia, lack of hair glossiness, increased lordokyphosis, periophthalmic lesions, and systemic senile amyloidosis [23]. These mice also displayed cognitive and behavioral alterations that were accompanied by molecular features typical of Alzheimer's disease (AD), such as overproduction of amyloid-beta protein, increased tau phosphorylation, cholinergic deficits in the forebrain and increased oxidative stress [18,19,24-28]. Biomarkers of inflammation such as $\mathrm{C}$-reactive protein and serum amyloid $\mathrm{P}$ are elevated in P8 mice [29].

Here we found that P8 mice present a spontaneous decrease in Rcor 2 gene expression, which is, at least in part, responsible for the inflamed phenotype in astrocytes. Our data show that Rcor 2 downregulation and neuroinflammation are mutually influenced processes.

\section{Materials and methods}

Animal care

All experimental procedures were approved by the Ethics Committee of the Autonomous University of Barcelona
(Comissió Ètica d'Experimentació Animal i Humana, CEEAH, UAB), following the 'Principles of laboratory animal care', and were performed in accordance with the European Communities Council Directive (86/609/EEC). SAMP8 and SAMR1 female mice were provided by the Parc Cientific, (University of Barcelona, Spain) and were maintained under standard conditions (temperature $23 \pm$ $1{ }^{\circ} \mathrm{C}$, humidity 50 to $60 \%, 12: 12$-h light-dark cycle, lights on at 7:00 a.m.), with food (A04, Harlan, Spain) and tap water available ad libitum throughout the study. Body weight (g) was measured weekly. For tissue harvesting, mice were sacrificed by cervical dislocation. The hippocampi and cerebral cortices were immediately frozen and stored at $-80^{\circ} \mathrm{C}$ for further analysis. The spleen was extracted and sliced, and splenocytes were isolated through a $70-\mu \mathrm{m}$ cell strainer (BD Biosciences, San Jose, CA) in PBS $1 \mathrm{X}$ and collected following centrifugation at $1600 \mathrm{rpm}$ for 5 minutes. Erythrocytes were further lysed using BD Pharm Lyse ${ }^{\mathrm{Tm}}$ solution (BD Biosciences, San Jose, $\mathrm{CA})$ at $37^{\circ} \mathrm{C}$ for $2 \mathrm{~min}$. Cells were recovered by centrifugation and pellets were aliquoted and frozen at $-80^{\circ} \mathrm{C}$ for further analysis.

Lipopolysaccharide treatment and cytokine determination SAMP8 and SAMR1 mice (males, 12-months old, $\mathrm{n}=3$ / group) received an intraperitoneal (IP) injection of lipopolysaccharide (LPS) $(2.9 \mathrm{mg} / \mathrm{kg}$; Sigma-Aldrich, St. Louis, Mo., USA), or an equal volume of saline. Three hours after treatment, blood samples were collected and mice were sacrificed by cervical dislocation. Tissues were collected and immediately frozen in liquid nitrogen and stored at $-80^{\circ} \mathrm{C}$ until processed.

Detection of TNF- $\alpha$, IL1- $\beta$ and IL6 in plasma was performed by enzyme-linked immunosorbent assay, in accordance with the protocol provided by the manufacturer (eBioscience, San Diego, CA; R\&D Systems, Minneapolis, $\mathrm{MN}$ and Diaclone, Besançon, France respectively).

\section{Cell culture and transfection}

Primary cultures of astrocytes were established from cerebral cortical tissue of 2-day-old SAMR1 and SAMP8, as previously described [30]. Cortices were promptly dissected free of the meninges, diced into small cubes, and dissociated by incubation for 25 min with a $0.25 \%$ trypsin/ $1 \mathrm{mM}$ EDTA solution (Gibco-Invitrogen, Carlsbad, CA). After a further mechanical dissociation, cells were resuspended in Dulbecco's Modified Eagle's Medium supplemented with $2.5 \mathrm{mM}$ glutamine, $100 \mathrm{~g} / \mathrm{mL}$ gentamycin, and 20\% FBS (Gibco-Invitrogen, Carlsbad, CA). Cells were seeded at $5 \times 10^{4} \mathrm{cell} / \mathrm{cm}^{2}$ in T75 flasks and incubated at $37^{\circ} \mathrm{C}$ in a humidified atmosphere of $5 \% \mathrm{CO}_{2}-95 \%$ air. The culture medium was changed twice a week. The concentration of FBS was changed to $15 \%$ and $10 \%$ after 1 and 2 weeks of culture, respectively. After 3 weeks, the 
flasks were shaken in an orbital shaker at $200 \mathrm{rpm}$ for $4 \mathrm{~h}$ to dislodge microglia and, subsequently, the attached astrocyte monolayer was collected. This purified fraction contained more than 90 to $95 \%$ of astrocytes, with a minor fraction of contaminating microglia.

Astrocytes were trypsinized $1 \mathrm{~h}$ before transfection, distributed into 48 -well plates $\left(1 \times 10^{5}\right.$ cells/well $)$ and subsequently transfected with $60 \mathrm{nM}$ of double-stranded siRNAs using Metafectene Pro (Biontex, Martinsried, Germany) at a 1:1 ratio with the siRNA. The sequences of the siRNAs used were: 5'-GGUCUUGACUCACAGCU CAtt-3' (Sense strand) and 5'-UGAGCUGUGAGUCAA GACCtc-3' (Antisense strand) for siRCOR2; and Silencer ${ }^{\text {TM }}$ negative control siRNA \#1 (Applied Biosystems, Foster City, CA) for siC. RNA was extracted $48 \mathrm{~h}$ after transfection.

Rat C6 glioma cells (purchased from the European Collection of Cell Cultures, ECACC) were cultured as monolayer and grown in F12/DMEM medium (Gibco-Invitrogen, Carlsbad, CA) supplemented with 10\% fetal bovine serum (Gibco-Invitrogen, Carlsbad, CA), 1\% non essential a/a's (Gibco-Invitrogen, Carlsbad, CA), 1/500 gentamicin (Gibco-Invitrogen, Carlsbad, CA) and maintained at $37^{\circ} \mathrm{C}$ and $5 \% \mathrm{CO}_{2}$ in a humidified environment.

For each treatment, C6 cells were seeded in a six-well plate and cultured in F12/DMEM without serum for $12 \mathrm{~h}$ prior to stimulation with LPS. Cells were incubated with LPS $(1 \mu \mathrm{g} / \mathrm{ml})$ for different time periods ranging from $30 \mathrm{~min}$ to $6 \mathrm{~h}$.

\section{Total RNA extraction}

Total RNA was extracted from frozen tissues through mirVana $^{\mathrm{m}}$ RNA Isolation Kit (Applied Biosystems, Foster City, CA) in accordance with the manufacturer's instructions.

RNA extraction from astrocytes and C6 cultured cells was performed using TRIzol $^{\mathrm{TM}}$ Reagent (Invitrogen, Carlsbad, CA) in accordance with the manufacturer's protocol.

The yield, purity, and quality of RNA were determined spectrophotometrically (Nano-drop Technologies, Wilmington, DE) and using the Bioanalyzer 2100 capillary electrophoresis (Agilent Technologies, Palo Alto, CA).

RNA samples with a 260/280 ratio and an RIN higher than 1.9 and 7.5, respectively, were selected.

\section{Real-time quantitative polymerase chain reaction}

Random-primed cDNA synthesis was performed at $37^{\circ} \mathrm{C}$ starting with $0.3 \mu \mathrm{g}$ of RNA, using the High Capacity cDNA Archive kit (Applied Biosystems, Foster City, CA). Gene expression was measured in an ABI Prism 7900HT Real Time PCR system using TaqMan FAM labeled specific probes (Applied Biosystems, , Foster City, CA [see
Additional file 1]). Results were normalized to the housekeeping TATA-binding protein $(T b p)$ gene.

\section{Statistical analysis}

The statistical analysis was carried out using the Statistical Package for Social Sciences (SPSS, version 19.0, www.ibm. com/software/es/analytics/spss/). An analysis of variance (ANOVA) was conducted to assess the effects of strain and specific conditions. Comparisons between groups were performed by the two-tailed Student's t-test for independent samples or the Mann-Whitney U-test as indicated.

Partial correlation (controlling for time of exposure to LPS) was used to examine the relationship between $\mathrm{Il}-6$ and Rcor 2 expression in the C6 glial cell line.

All values were expressed as mean \pm standard error of the mean. $P<0.05$ was considered significant. Statistical outliers ( $\geq$ two standard deviations from the mean) were removed from analyses.

\section{Results and discussion}

Increased levels of IL6 in serum of P8 mice after intraperitoneal lipopolysaccharide administration

P8 senescent mice exhibited signs of systemic inflammation affecting the liver, pancreas, cochlea, bones, testes and plasma levels of several cytokines and proinflammatory factors [29,31-37]. In addition to their peripheral inflamed phenotype, a proinflammatory brain environment may be involved in the neurodegenerative traits and/or cognitive deficiency in P8 mice [38]. The inflammatory response was analyzed three hours after LPS intraperitoneal injection in 12 month-old P8 mice and age-matched R1 controls. A pronounced increase was found in IL1 $\beta$, TNF $\alpha$ and IL6 serum levels both in P8 and R1 mice in response to LPS (IL1 $\beta$ : $\mathrm{F}(1,7)=0.962, P<0.05$; TNF $\alpha$ : $\mathrm{H}(3)=8.389$, $P<0.05$; IL6: $\mathrm{F}(1,7)=67.362, P<0.001)$ (Figure $1 \mathrm{~A}$ and $\mathrm{B}$ ). Neither strain effect nor interaction between strain and LPS treatment was observed for IL1 $\beta$ and TNF $\alpha$ cytokines. In contrast, we found both strain and LPS effects for IL6 $(\mathrm{F}(1,7)=12.706, P<0.01 ; \mathrm{F}(1,7)=67.362, P<0.001)$ as well as a strong tendency for interaction between strain and treatment $(P=0.068)$. Moreover, IL6 showed increased basal levels in P8 mice $(\mathrm{t}(3)=-4.139 ; P<0.05)$ (Figure $1 \mathrm{C}$ ). These data suggest that plasma IL6 could be an inflammatory biomarker of P8 inflamed phenotype.

\section{Decreased Rcor2 expression in P8 splenocytes, cortex, hippocampus and astrocytes}

We explored the expression of Rcor 1 and Rcor2 in P8 mice with the aim of elucidating a possible mechanism for their inflamed phenotype and increased response to LPS treatment. We analyzed the Rcor gene expression pattern in splenocytes from P8 and R1 mice at three different ages: 7 days, 3 months and 9 months. Both strains 


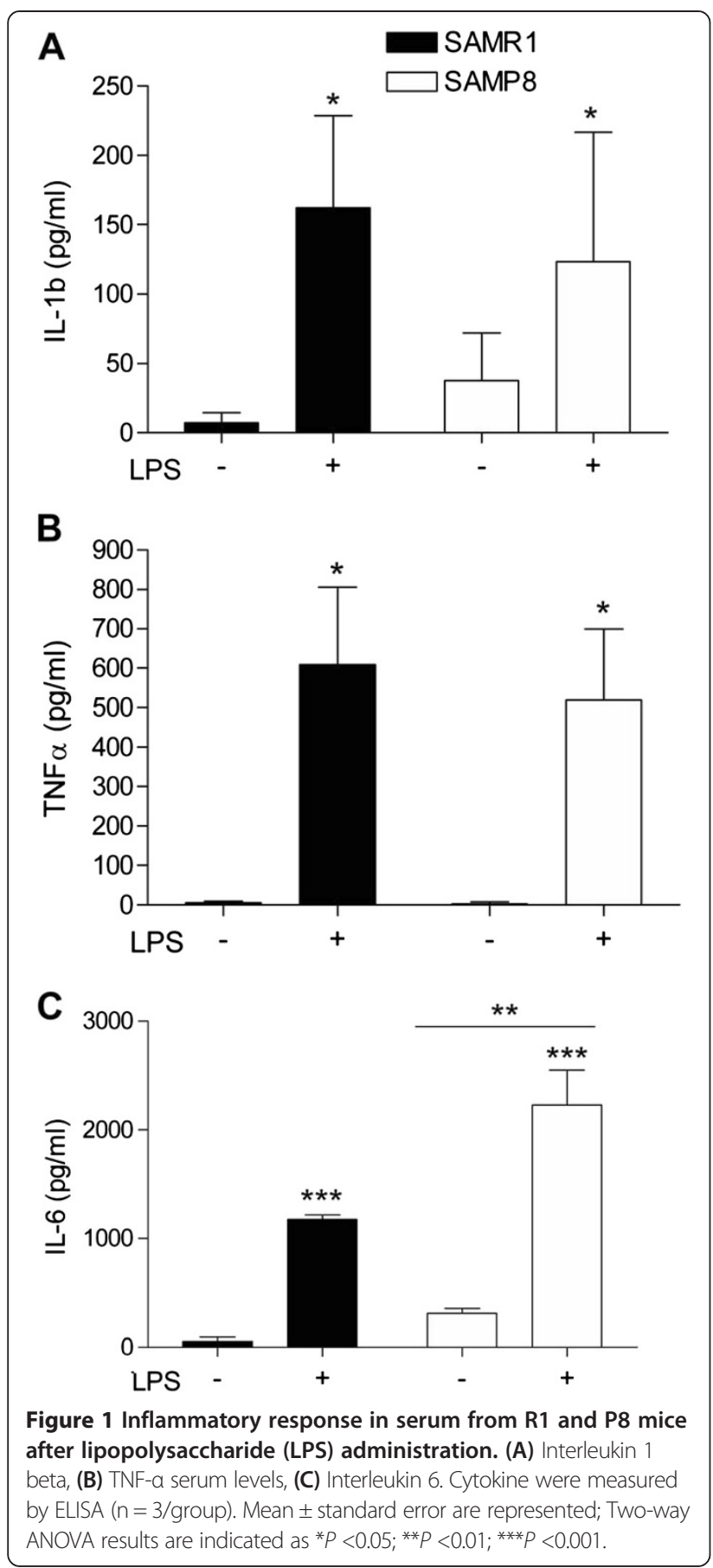

presented similar age-dependent gene expression patterns for Rcor1 with higher levels at 7 days and 9 months compared with 3 months $(\mathrm{F}(2,22)=32.306, P<0.001)$ (Figure 2A). In contrast, R1 mice showed an agedependent increase in Rcor2 gene expression which was not observed in P8 mice (interaction between strain and age: $\mathrm{F}(2,29)=4.279, P<0.05$ ) (Figure $2 \mathrm{~B}$ ). Intriguingly, $\mathrm{P} 8$ mice exhibited low expression levels of Rcor2 from the first week of age and throughout the adult life. These data

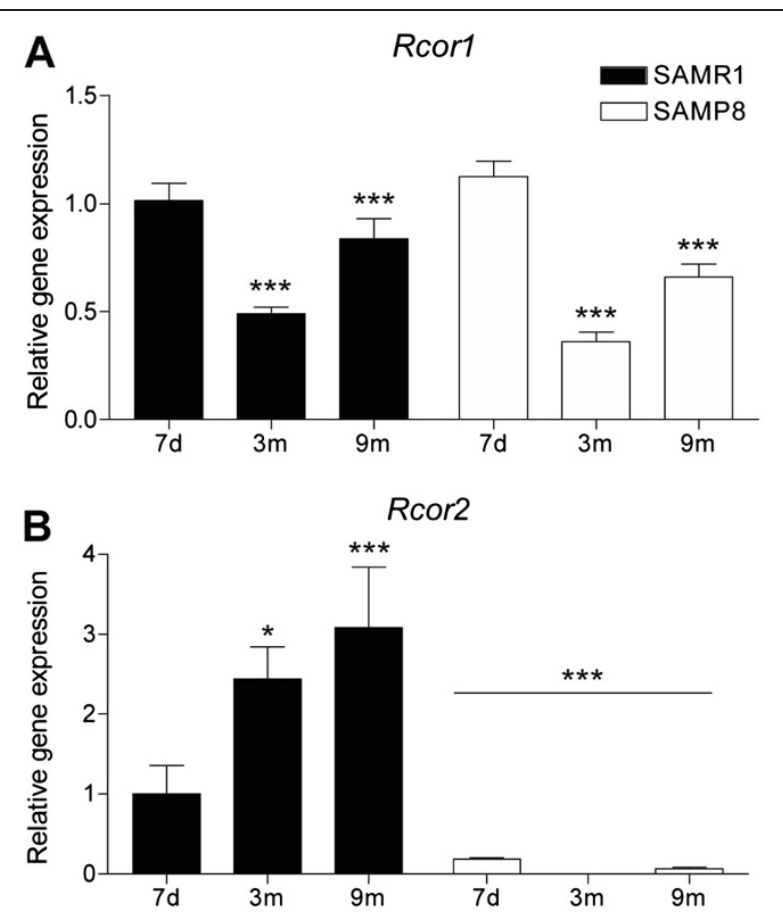

Figure 2 Rcor gene expression profile at different ages in splenocytes from R1 and P8 mice. (A) Rcor1 and (B) Rcor2 gene expression at the ages of 7 days, 3 months and 9 months ( $n=3$ to 10/group). Gene expression levels were determined by real time PCR using TaqMan FAM-labeled specific probes and expressed relative to Tbp. Mean \pm standard error are represented; Two-way ANOVA results are indicated as ${ }^{*} P<0.05 ;{ }^{* *} P<0.001$.

indicate that Rcor2 underexpression in splenocytes precedes most of the accelerated-aging characteristics of this model. Indeed, we found a marked effect for strain and age in Rcor2 gene expression $(\mathrm{F}(1,29)=31.620, P<0.001$; $\mathrm{F}(2,29)=3.363, P<0.001$; respectively $)$.

Chronic inflammation is associated with early development and acceleration of neurodegenerative diseases such as Alzheimer's disease [39,40]. Therefore, we analyzed the expression of Rcor 1 and Rcor2 in P8 cortices and hippocampi. Rcor1 presented age effect in both cortex and hippocampus tissues $(\mathrm{F}(2,27)=49.140, P<0.001 ; \mathrm{F}(2,32)=12.597$, $P<0.001$; respectively) with a similar age-dependent expression pattern in both strains (Figure $3 \mathrm{~A}$ and B). However, 9-month-old P8 mice, unlike R1 mice, presented slight but significant alterations in Rcor 1 levels in the cortex and in the hippocampus. On the other hand, Rcor2 was expressed similarly in R1 and P8 brain cortices and hippocampi at 7 days and 3 months of age (Figure 3C and D).

Remarkably, a consistent characteristic among splenocytes, cortex and hippocampus was that Rcor 2 gene expression in 9-month-old P8 mice decreased significantly in comparison with $\mathrm{R} 1$ controls (cortex: $P<0.001$; hippocampus: $P<0.01)$. 

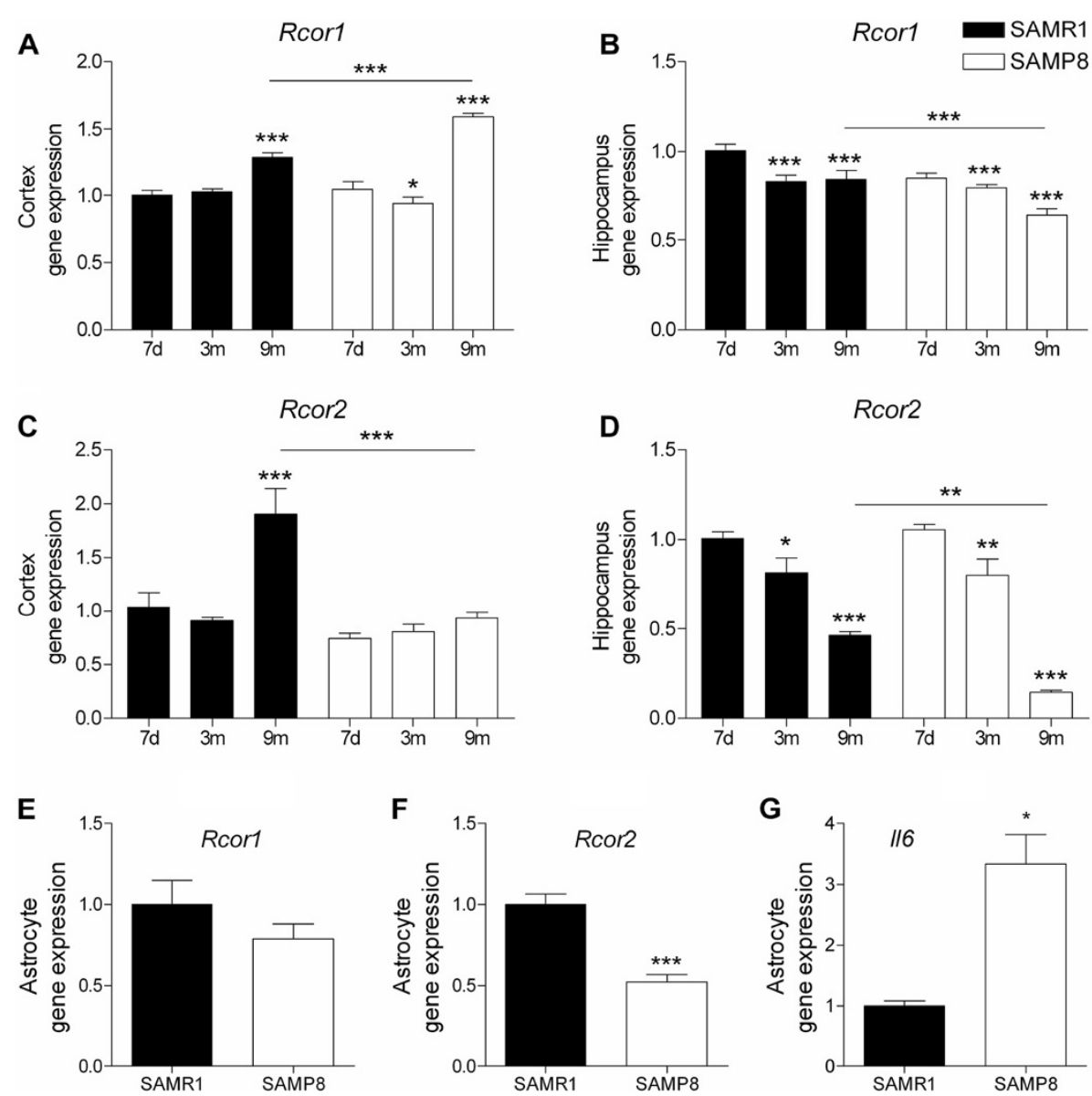

Figure 3 Rcor gene expression profile in brain tissues from R1 and P8 mice. Rcor1 and Rcor2 gene expression was quantified in the cortex ( $A$ and $\mathbf{C}$ ), in the hippocampus ( $B$ and $\mathbf{D}$ ) at the ages 7 days, 3 months and 9 months ( $n=4$ to 8/group), and in primary cultured astrocytes ( $\mathrm{n}=6$ to 9/group) (E and F) from 2 days-old mice. (G) Interleukin 6 basal gene expression levels in primary cultured astrocytes obtained from 2-day-old mice $(n=4 /$ group). Gene expression levels were measured by real time PCR. Mean \pm standard error are represented; Two-way ANOVA results are indicated as ${ }^{*} P<0.05 ;{ }^{* *} P<0.01 ;{ }^{* *} P<0.001$.

We also explored the gene expression of Rcor1 and Rcor2 in P8 and R1 astrocytes obtained from cerebral cortical tissue of 2-day-old mice. P8 cells showed similar Rcor1 but decreased Rcor 2 gene expression in comparison with R1 cells ( $\mathrm{t}(11)=5,837 ; P<0.001)$ (Figure 3E and F). Notably, the reduced levels of Rcor2 in P8 compared with R1 astrocytes were accompanied by increased basal levels of Il6 gene expression $(\mathrm{t}(3)=-4.734$; $P=0.016)$ (Figure 3G).

These data show that Rcor2 is underexpressed in different tissues and organs in P8 mice and, in some cases (namely, in splenocytes and astrocytes), this alteration is detected from early ages. Interestingly, Yankner and colleagues have recently shown that the transcriptional repressor REST (RE1-silencing transcription factor) is crucial for neuroprotection during aging [14]. It is known that REST recruitment of RCOR corepressor is required for its gene silencing activity [15]. The downregulation of Rcor2 in immune and brain cells of the P8 senescent mice strongly suggests that the accelerated aging in these mice, as well as their neurodegenerative phenotype, may be related to Rcor2 deficiency.

On the other hand, RCOR is part of a chromatin remodeling complex containing the lysine specific demethylase (KDM1A), which catalyzes $\mathrm{H} 3$ histone demethylation as a way to repress transcription [12]. In the absence of RCOR, KDM1A demethylase activity is impaired with a concomitant increase in H3 histone methylation levels. In this context, preliminary data from our group indicate that the alteration in histone methylation levels is a candidate mechanism through which Rcor 2 could also influence the aging process in P8 mice. Indeed, P8 mice present increased levels of trimethylated histone $\mathrm{H} 3$ lysine 4 (H3K4me3) in splenocytes, cortex, and hippocampus (supplementary information, [see Additional file 2A-C]). Moreover, consistent with a role of Rcor in methylation signaling and in neuroinflammation, we have found that Il6 upregulation in response to LPS is significantly 
dependent on methyltransferase activity (supplementary information, [see Additional file 3]).

\section{Negative correlation between Rcor2 and inflammatory} gene expression after lipopolysaccharide (LPS) treatment in $\mathrm{C} 6$ glioma cells and mouse hippocampus

To approach the possible role of Rcor2 in neuroinflammation, we first analyzed its gene expression after an in vivo intraperitoneal LPS injection in 12-month-old P8 and R1 mice. While P8 hippocampus was not further responsive to the peripheral LPS treatment [see Additional file 4], R1 mice presented a significant decrease in Rcor2 expression $(\mathrm{t}(12)=2.606, P=0.023)$ together with an increase in $\operatorname{Tnf} \alpha$, $I l 1 \beta$ and Il6 gene expression levels $(\mathrm{t}(5.01)=-2.726$, $P<0.05 ; \mathrm{t}(5.02)=-2.490, P=0.055 ; \mathrm{U}(9)=0.000, \mathrm{Z}=-2.449$, $P<0.05$; respectively) (Figure $4 \mathrm{~A}-\mathrm{D}$ ).

A similar effect was found in C6 glioma cells treated with LPS. C6 cells were activated with LPS for $30 \mathrm{~min}$ and $1,2,3$ and 6 hours. We found a significant reduction in
Rcor2 gene expression $(\mathrm{F}(5,12)=47.332 ; P<0.001)$ with a concomitant increase in $I l 6$ expression in response to LPS $(\mathrm{F}(5,11)=106.833 ; P<0.001$ ) (Figure $5 \mathrm{~A}$ and $\mathrm{B}$, respectively). A significant correlation (controlling for time of exposure to LPS) was found between $I L-6$ and Rcor2 expression ( $\rho r=-0.512$ and $P=0.043$ ) (Figure 5C).

\section{Knock down of Rcor2 in astrocytes upregulates $/ 16$ gene expression}

To determine the involvement of Rcor 2 in the modulation of Il6 expression, we knocked down Rcor2 in R1 and P8 astrocytes by transfection of a siRNA to Rcor2 (siRcor2) using a scrambled siRNA as a control (siC).

Rcor 2 knock down led to a $31 \%$ and $46 \%$ decrease in its expression levels in R1 and P8 astrocytes, respectively $(\mathrm{F}(1,7)=10.082, P=0.016)$ (Figure 6A). Moreover, siRcor2 astrocytes showed increased $I l 6$ levels in both strains with significantly higher Il6 levels in P8 compared with the R1 cells (strain: $\mathrm{F}(1,6)=10.022, P<0.05)$ (Figure 6B).
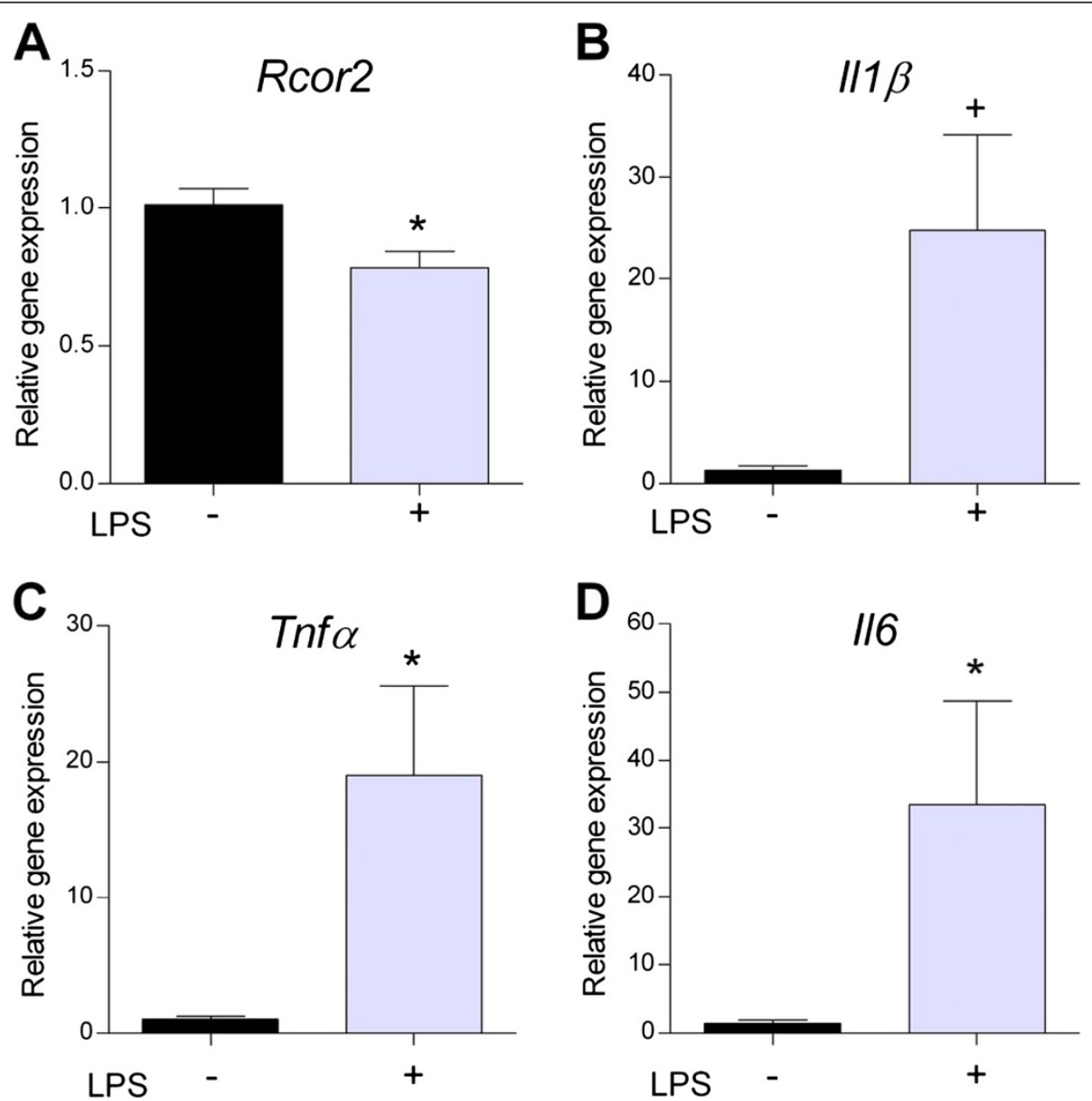

Figure 4 Concomitant downregulation of Rcor2 and increased pro-inflammatory gene expression in the R1 hippocampus after intraperitoneal lipopolysaccharide (LPS) injection. (A) Rcor2 (B) interleukin 1 beta, (C) tnf-alpha and) (D) interleukin 6 gene expression levels in hippocampus from 12 month-old R1 after intraperitoneal lipopolysaccharide (LPS) injection ( $n=5$ to $7 /$ group). Real-time PCR was performed and means \pm standard errors are represented. Two-way ANOVA results are indicated as $* P<0.05$. 
A

Rcor2

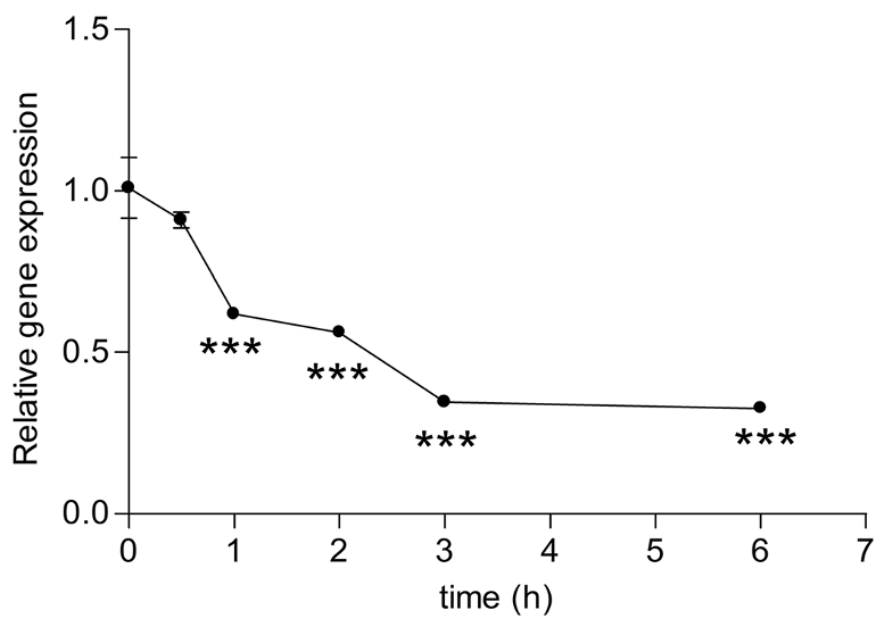

B

$1 / 6$
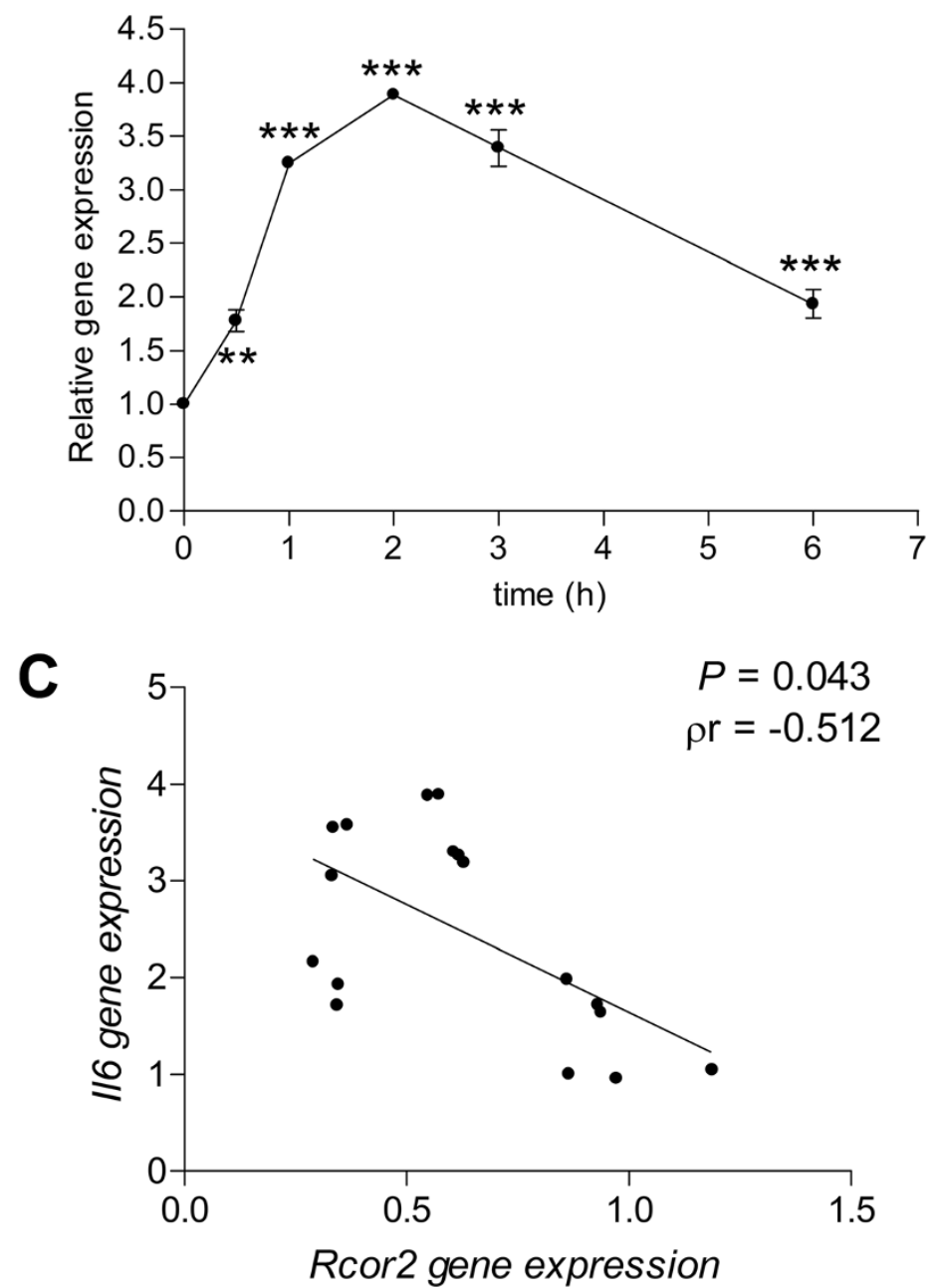

Figure $\mathbf{5}$ (See legend on next page.) 
(See figure on previous page.)

Figure 5 Concomitant downregulation of Rcor2 and increased IL6 gene expression in lipopolysaccharide (LPS)-treated C6 glial cell line. (A) Rcor2 and (B) interleukin 6 gene expression after LPS stimulation $(1 \mu \mathrm{g} / \mathrm{ml})$ in C6 glial cells. Gene expression levels were determined by real-time PCR. Mean \pm standard error from three independent experiments are represented. One-way ANOVA results are indicated as ${ }^{* *} P<0.01$; ${ }^{* * *} P<0.001$. (C) Partial correlation between Rcor2 and I/6 gene response after LPS treatment in C6 glial cells. P value (P) and partial correlation coefficient (pr) are indicated.

Knock-down data in R1 and P8 astrocytes reinforce the notion that Rcor gene product acts as a repressor of an inflammatory program [16,17]. Our findings suggest that Rcor 2 underexpression may be at the root of the inflamed phenotype that characterizes the P8 strain [29].

\section{Interplay between Rcor2 expression and inflammatory response in siRcor2 astrocytes}

Il6 gene expression was then analyzed in R1 and P8 astrocytes transfected with siRcor 2 following activation by LPS and Interferon gamma (IFNY). Astrocytes from both strains transfected with scrambled siRNA (siC) were used as controls.

Notably, LPS/IFNy treatment further reduced Rcor2 expression $(\mathrm{F}(1,14)=47.465, P<0.001)$ with a concomitant
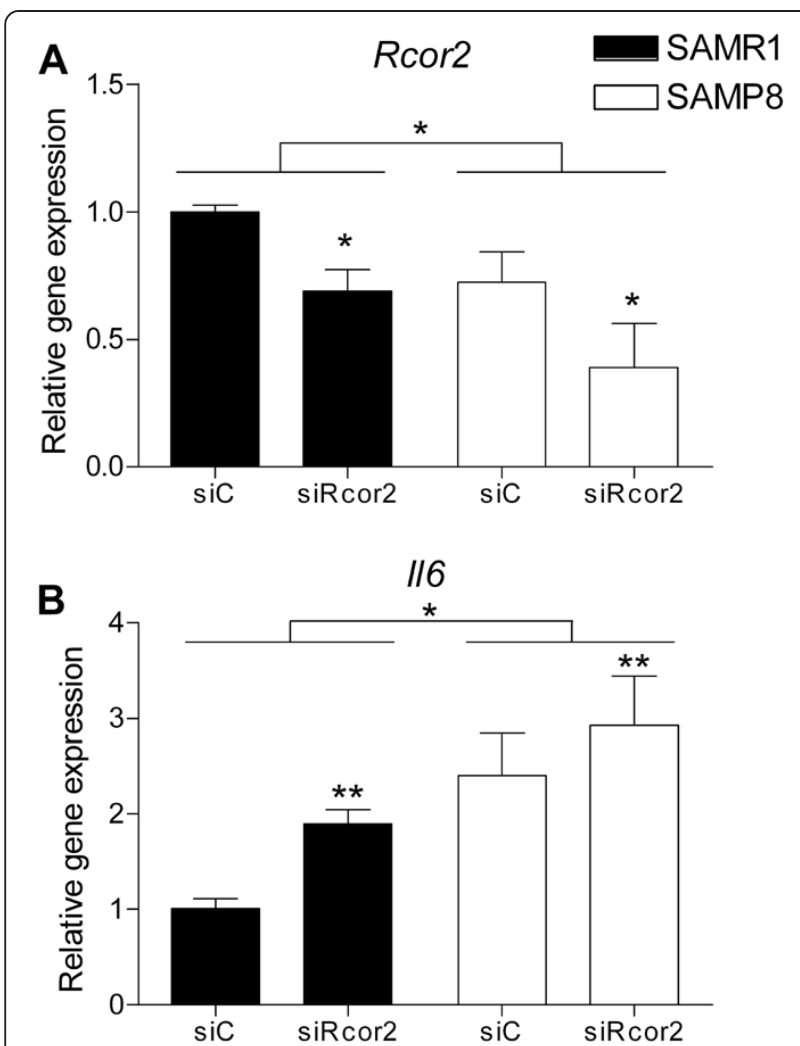

Figure 6 Knock down of Rcor 2 in P8 and R1 astrocytes.

(A) Rcor2 and (B) Interleukin 6 basal gene expression levels after transfection in P8 and R1 astrocytes of a siRNA to Rcor2 (siRcor2) using a scrambled siRNA as a control (siC). Gene expression levels were measured by real-time PCR. Mean \pm standard error from three independent experiments are represented; Two-way ANOVA results are indicated as ${ }^{*} P<0.05$. increase in $\mathrm{Il6}$ expression in all conditions tested ( $\mathrm{siC}$ and siRcos2 transfected $\mathrm{R} 1$ and P8 cells) $(\mathrm{F}(1,14)=134.730$, $P<0.001$ ) (Figure 7B) (Figure 7A and $\mathrm{B}$, a versus b; c versus d; f versus e; and g versus $h$ ).

For Rcor2, the interaction between strain and LPS/ IFN $\gamma$ stimulation was also significant $(\mathrm{F}(1,14)=16.673$, $P<0.01)$ indicating that the treatment led to a greater decrease in Rcor 2 after LPS/IFNy stimulation in P8 than in R1 cells. Il6 expression in response to the stimulus was significantly exacerbated in siRcor2 knock down astrocytes in comparison with siC-transfected cells (transfection $\mathrm{X}$ treatment effect: $\mathrm{F}(1,14)=11.311, P<0.01$ ) (Figure 7B, d versus b and h versus $\mathrm{f}$ ).

These results suggest that Rcor 2 downregulation in astrocytes induces inflammation and in turn, an

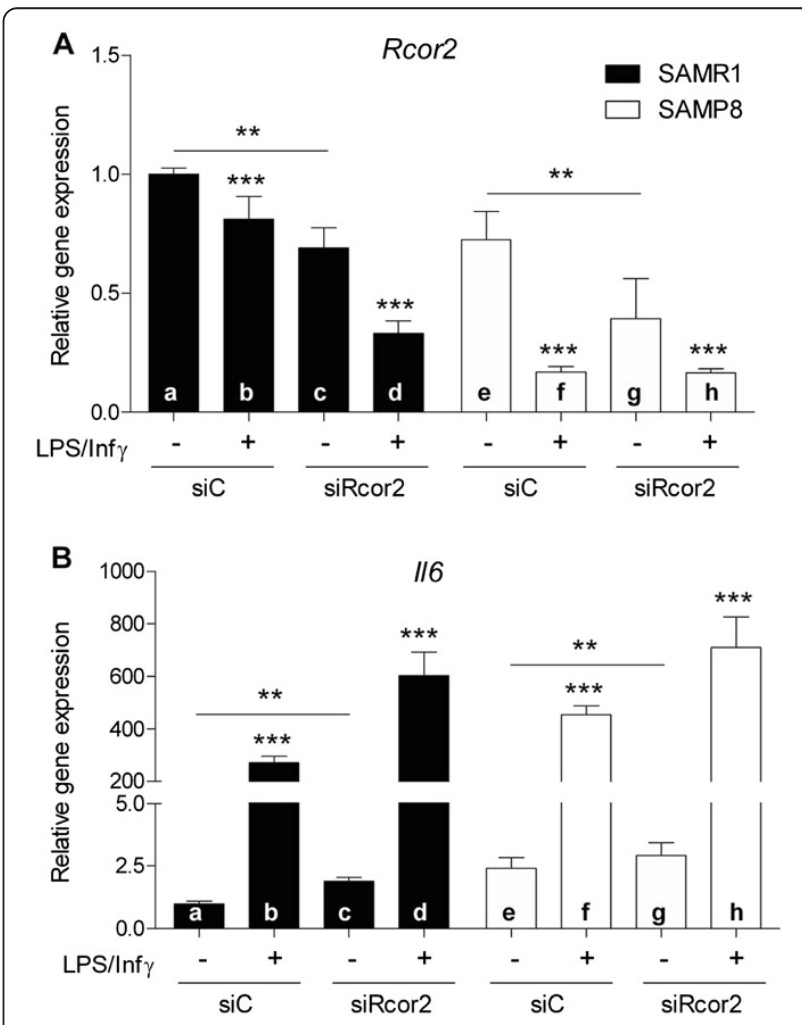

Figure 7 Interplay between Rcor2 expression and inflammatory response in siRcor2 astrocytes. (A) Rcor2 and (B) interleukin 6 gene expression levels after $4.5 \mathrm{~h}$ lipopolysaccharide (LPS) $(2 \mathrm{\mu g} / \mathrm{ml})$ and interferon-gamma $(3 \mu \mathrm{g} / \mathrm{ml})$ stimulation in R1 and P8 astrocytes transfected with siRNA to Rcor2 (siRcor2) or scrambled siRNA as a control (siC) ( $n=3 /$ group). Gene expression levels were determined by real-time PCR. Mean \pm standard error are represented; Two-way ANOVA results are indicated as ${ }^{* *} P<0.01 ;{ }^{* * *} P<0.001$. 
inflammatory environment further downregulates Rcor2. We can conclude from our data on transfected astrocytes that the LPS/INFy stimulus and Rcor2 knock down represent independent modulators of 16 expression and show a synergic effect when combined.

\section{Conclusions}

Little information is currently available on the molecular triggers of chronic low-grade inflammation, a process that affects the function of most organs and tissues including the central nervous system and is involved in aging and neurodegeneration [41,42].

RCOR is found in a complex with RE1-silencing transcription factor (REST), which plays a role in protecting the brain from age-related insults in animal models and a role in preservation of cognitive function and longevity in aging humans [14]. Taking into account the early downregulation of RCOR2 in the P8 mouse model of accelerated aging and neurodegeneration, further studies are warranted so as to examine whether the REST/ RCOR complex is involved in the shorter lifespan of P8 mice as well as the role of RCOR2 in human cognition and longevity.

Our study indicates that neuroinflammation is a pathophysiological outcome of Rcor2 downregulation in the SAMP8 mice. The fact that Rcor2 and Il6 presented an opposite response after an inflammatory stimulus in hippocampus and in glial cells, suggests a possible interplay between the pathways involved in the regulation of both genes. This notion is reinforced by our data showing that $I l 6$ gene is upregulated by Rcor 2 knock down in astrocytes. Therefore, RCOR2 may represent a potential target for the prevention or treatment of age-related chronic inflammation and neurodegeneration.

\section{Additional files}

Additional file 1: List of primers and probe sets used for real time RT-PCR analysis.

Additional file 2: Histone $\mathrm{H} 3$ methylation pattern in $\mathrm{R} 1$ and $\mathrm{P} 8$ tissues.

Additional file 3: Methyltranferase inhibitor MTA reduces lipopolysaccharide (LPS)-induced Il6 gene expression in C6 glioma cells.

Additional file 4: Pro-inflammatory gene expression response in P8 hippocampus after intraperitoneal lipopolysaccharide (LPS) injection.

\section{Abbreviations}

Rcor: REST corepressor; P8: senescence-accelerated prone 8 mouse; R1: senescence-accelerated resistant 1; LPS: lipopolysaccharide; L: interleukin; Tnf-a: tumor necrosis factor; AD: Alzheimer's disease; KDM1A: histone demethylase lysine-specific demethylase 1A; REST: RE1-silencing transcription factor; ESCs: embryonic stem cells; CCI2: chemokine ligand 2; Mip 1a: macrophage proinflammatory protein 1a; CXCl: chemokine (C-X-C motif) ligands; NF-KB: nuclear factor kappa B; IFNy: Interferon gamma; Rcor: REST corepressor; P8: senescence-accelerated prone 8 mouse;
R1: senescence-accelerated resistant 1; LPS: lipopolysaccharide; L: interleukin; Tnf-a: tumor necrosis factor; AD: Alzheimer's disease; KDM1A: histone demethylase lysine-specific demethylase 1A; REST: RE1-silencing transcription factor; ESCs: embryonic stem cells; CCl2: chemokine ligand 2;

Mip 1a: macrophage proinflammatory protein 1a; $C x$ l: chemokine (C-X-C motif) ligands; NF-kB: nuclear factor kappa B; IFNy: Interferon gamma.

\section{Competing interests}

The authors declare that they have no competing interests.

\section{Authors' contributions}

Designed research: MJAL and PK; performed research: MJAL, PMM, MCF, MCT, MPar, RC, RME, MP and PK; contributed new reagents/analytic tools: MPar, RME, MP, CS and PK; analyzed data MJAL, MCF, MCT, MPar, RC, CS, and PK; wrote the manuscript: MJAL and PK. All authors read and approved the final manuscript.

\section{Author details}

'Institut d'Investigacions Biomèdiques de Barcelona (IIBB), CSIC, Rosselló 161, E-08036 Barcelona, Spain. ${ }^{2}$ Unidad de Farmacología y Farmacognósia. Institut de Biomedicina (IBUB), Universidad de Barcelona y CIBERNED. Facultad de Farmacia, Av Diagonal, 643, E-08028 Barcelona, Spain. ${ }^{3}$ Instituto de Investigaciones Biomédicas August Pi i Sunyer (IDIBAPS), Rosellón 149 E-08036 Barcelona, Spain. ${ }^{4}$ Instituto de Neurociencias, Departamento de Psiquiatría y Medicina Legal. Facultad de Medicina, Universitat Autònoma de Barcelona, Campus de Bellaterra, Bellaterra (Cerdanyola del Vallès), E-08193 Barcelona, Spain.

Received: 15 April 2014 Accepted: 7 July 2014

Published: 23 July 2014

\section{References}

1. Franceschi C, Bonafe M, Valensin S, Olivieri F, De Luca M, Ottaviani E, De Benedictis G: Inflamm-aging: an evolutionary perspective on immunosenescence. Ann N Y Acad Sci 2000, 908:244-254.

2. Cevenini E, Monti D, Franceschi C: Inflamm-ageing. Curr Opin Clin Nutr Metab Care 2013, 16:14-20.

3. Vasto S, Candore G, Balistreri CR, Caruso M, Colonna-Romano G, Grimaldi MP, Listi F, Nuzzo D, Lio D, Caruso C: Inflammatory networks in ageing, age-related diseases and longevity. Mech Ageing Dev 2007, 128:83-91.

4. Kaliman P, Parrizas M: Obesity and systemic inflammation: insight on epigenetic mechanisms. In Role of the Adipocyte in the Development of Type 2 Diabetes, Volume 1st. Edited by Rijeka CC. Croatia: InTech; 2011:65-88.

5. Pizza V, Agresta A, D'Acunto CW, Festa M, Capasso A: Neuroinflammation and ageing: current theories and an overview of the data. Rev Recent Clin Trials 2011, 6:189-203.

6. Liu Y, Zhang M, Hao W, Mihaljevic I, Liu X, Xie K, Walter S, Fassbender K: Matrix metalloproteinase-12 contributes to neuroinflammation in the aged brain. Neurobiol Aging 2013, 34:1231-1239.

7. McGeer PL, McGeer EG: Inflammation and the degenerative diseases of aging. Ann N Y Acad Sci 2004, 1035:104-116.

8. Lee M: Neurotransmitters and microglial-mediated neuroinflammation. Curr Protein Pept Sci 2013, 14:21-32.

9. Bardou I, Brothers HM, Kaercher RM, Hopp SC, Wenk GL: Differential effects of duration and age on the consequences of neuroinflammation in the hippocampus. Neurobiol Aging 2013, 34:2293-2301.

10. Farso M, Menard C, Colby-Milley J, Quirion R: Immune marker CD68 correlates with cognitive impairment in normally aged rats. Neurobiol Aging 2013, 34:1971-1976.

11. Lee MG, Wynder C, Cooch N, Shiekhattar R: An essential role for CoREST in nucleosomal histone 3 lysine 4 demethylation. Nature 2005, 437:432-435.

12. Shi Y, Lan F, Matson C, Mulligan P, Whetstine JR, Cole PA, Casero RA, Shi Y: Histone demethylation mediated by the nuclear amine oxidase homolog LSD1. Cell 2004, 119:941-953.

13. Yang P, Wang Y, Chen J, Li H, Kang L, Zhang Y, Chen S, Zhu B, Gao S: RCOR2 is a subunit of the LSD1 complex that regulates ESC property and substitutes for SOX2 in reprogramming somatic cells to pluripotency. Stem Cells 2011, 29:791-801. 
14. Lu T, Aron L, Zullo J, Pan Y, Kim H, Chen Y, Yang TH, Kim HM, Drake D, Liu XS, Bennett DA, Colaiácovo MP, Yankner BA: REST and stress resistance in ageing and Alzheimer's disease. Nature 2014, 507:448-454.

15. Andres ME, Burger C, Peral-Rubio MJ, Battaglioli E, Anderson ME, Grimes J, Dallman J, Ballas N, Mandel G: CoREST: a functional corepressor required for regulation of neural-specific gene expression. Proc Natl Acad Sci U S A 1999, 96:9873-9878.

16. Hanzu FA, Musri MM, Sanchez-Herrero A, Claret M, Esteban Y, Kaliman P, Gomis $R$, Parrizas M: Histone demethylase KDM1A represses inflammatory gene expression in preadipocytes. Obesity (Silver Spring) 2013, 21:E616-E625.

17. Saijo K, Winner B, Carson CT, Collier JG, Boyer L, Rosenfeld MG, Gage FH, Glass CK: A Nurr1/CoREST pathway in microglia and astrocytes protects dopaminergic neurons from inflammation-induced death. Cell 2009, 137:47-59.

18. Cheng XR, Zhou WX, Zhang YX: The behavioral, pathological and therapeutic features of the senescence-accelerated mouse prone 8 strain as an Alzheimer's disease animal model. Ageing Res Rev 2014, $13: 13-37$

19. Morley JE, Farr SA, Kumar VB, Armbrecht HJ: The SAMP8 mouse: a model to develop therapeutic interventions for Alzheimer's disease. Curr Pharm Des 2012, 18:1123-1130.

20. Pallas M, Camins A, Smith MA, Perry G, Lee HG, Casadesus G: From aging to Alzheimer's disease: unveiling "the switch" with the senescenceaccelerated mouse model (SAMP8). J Alzheimers Dis 2008, 15:615-624.

21. Wang H, Lian K, Han B, Wang Y, Kuo SH, Geng Y, Qiang J, Sun M, Wang M: Age-related alterations in the metabolic profile in the hippocampus of the senescence-accelerated mouse prone 8: a spontaneous Alzheimer's disease mouse model. J Alzheimers Dis 2014, 39:841-848.

22. Takeda T, Hosokawa M, Higuchi K: Senescence-accelerated mouse (SAM): a novel murine model of accelerated senescence. J Am Geriatr Soc 1991 39:911-919.

23. Nomura Y, Okuma Y: Age-related defects in lifespan and learning ability in SAMP8 mice. Neurobiol Aging 1999, 20:111-115.

24. Morley JE, Armbrecht HJ, Farr SA, Kumar VB: The senescence accelerated mouse (SAMP8) as a model for oxidative stress and Alzheimer's disease. Biochim Biophys Acta 2012, 1822:650-656.

25. del Valle J, Duran-Vilaregut J, Manich G, Pallas M, Camins A, Vilaplana J, Pelegri C: Cerebral amyloid angiopathy, blood-brain barrier disruption and amyloid accumulation in SAMP8 mice. Neurodegener Dis 2011 8:421-429.

26. Takeda T: Senescence-accelerated mouse (SAM) with special references to neurodegeneration models, SAMP8 and SAMP10 mice. Neurochem Res 2009, 34:639-659.

27. Canudas AM, Gutierrez-Cuesta J, Rodriguez MI, Acuna-Castroviejo D, Sureda FX, Camins A, Pallas M: Hyperphosphorylation of microtubule-associated protein tau in senescence-accelerated mouse (SAM). Mech Ageing Dev 2005, 126:1300-1304.

28. Sureda FX, Gutierrez-Cuesta J, Romeu M, Mulero M, Canudas AM, Camins A, Mallol J, Pallas M: Changes in oxidative stress parameters and neurodegeneration markers in the brain of the senescence-accelerated mice SAMP-8. Exp Gerontol 2006, 41:360-367.

29. Bayram B, Nikolai S, Huebbe P, Ozcelik B, Grimm S, Grune T, Frank J, Rimbach G: Biomarkers of oxidative stress, antioxidant defence and inflammation are altered in the senescence-accelerated mouse prone 8 . Age 2013, 35:1205-1217.

30. Garcia-Matas S, Gutierrez-Cuesta J, Coto-Montes A, Rubio-Acero R, Diez-Vives C, Camins A, Pallas M, Sanfeliu C, Cristofol R: Dysfunction of astrocytes in senescence-accelerated mice SAMP8 reduces their neuroprotective capacity. Aging Cell 2008, 7:630-640

31. Cuesta S, Kireev R, Forman K, Garcia C, Escames G, Ariznavarreta C, Vara E, Tresguerres JA: Melatonin improves inflammation processes in liver of senescence-accelerated prone male mice (SAMP8). Exp Gerontol 2010, 45:950-956

32. Cuesta S, Kireev R, Garcia C, Forman K, Escames G, Vara E, Tresguerres JA Beneficial effect of melatonin treatment on inflammation, apoptosis and oxidative stress on pancreas of a senescence accelerated mice model. Mech Ageing Dev 2011, 132:573-582.

33. Cuesta S, Kireev R, Garcia C, Forman K, Vara E, Tresquerres JA: Effect of growth hormone treatment on pancreatic inflammation, oxidative stress, and apoptosis related to aging in SAMP8 mice. Rejuvenation Res 2011, 14:501-512.
34. Menardo J, Tang Y, Ladrech S, Lenoir M, Casas F, Michel C, Bourien J, Ruel J, Rebillard G, Maurice T, Puel JL, Wang J: Oxidative stress, inflammation, and autophagic stress as the key mechanisms of premature age-related hearing loss in SAMP8 mouse cochlea. Antioxid Redox Signal 2012, 16:263-274

35. Rodriguez Ml, Escames G, Lopez LC, Lopez A, Garcia JA, Ortiz F, Acuna-Castroviejo $D$ : Chronic melatonin treatment reduces the age-dependent inflammatory process in senescence-accelerated mice. J Pineal Res 2007, 42:272-279.

36. Wauquier F, Barquissau V, Leotoing L, Davicco MJ, Lebecque P, Mercier S, Philippe C, Miot-Noirault E, Chardigny JM, Morio B, Wittrant Y, Coxam V: Borage and fish oils lifelong supplementation decreases inflammation and improves bone health in a murine model of senile osteoporosis. Bone 2012, 50:553-561.

37. Zhao X, Bian Y, Sun Y, Li L, Wang L, Zhao C, Shen Y, Song Q, Qu Y, Niu S, Wu W, Gao F: Effects of moderate exercise over different phases on age-related physiological dysfunction in testes of SAMP8 mice. Exp Gerontol 2013, 48:869-880.

38. Tha KK, Okuma Y, Miyazaki H, Murayama T, Uehara T, Hatakeyama R, Hayashi $Y$, Nomura $Y$ : Changes in expressions of proinflammatory cytokines IL-1 beta, TNF-alpha and IL- 6 in the brain of senescence accelerated mouse (SAM) P8. Brain Res 2000, 885:25-31.

39. Lyman M, Lloyd DG, Ji X, Vizcaychipi MP, Ma D: Neuroinflammation: the role and consequences. Neurosci Res 2014, 79C:1-12

40. Jana M, Palencia CA, Pahan K: Fibrillar amyloid-beta peptides activate microglia via TLR2: implications for Alzheimer's disease. J Immunol 2008, 181:7254-7262.

41. Yaffe K, Kanaya A, Lindquist K, Simonsick EM, Harris T, Shorr Rl, Tylavsky FA, Newman $A B$ : The metabolic syndrome, inflammation, and risk of cognitive decline. JAMA 2004, 292:2237-2242.

42. von Bernhardi R: Glial cell dysregulation: a new perspective on Alzheimer disease. Neurotox Res 2007, 12:215-232

doi:10.1186/1742-2094-11-126

Cite this article as: Alvarez-López et al:: Rcor2 underexpression in senescent mice: a target for inflammaging? Journal of Neuroinflammation 2014 11:126

\section{Submit your next manuscript to BioMed Central and take full advantage of:}

- Convenient online submission

- Thorough peer review

- No space constraints or color figure charges

- Immediate publication on acceptance

- Inclusion in PubMed, CAS, Scopus and Google Scholar

- Research which is freely available for redistribution 